\title{
NUMERICAL AND EXPERIMENTAL ANALYSIS OF QUENCH INDUCED STRESSES AND MICROSTRUCTURES
}

\author{
C. Hakan Gür ${ }^{1}$ and A. Erman Tekkaya ${ }^{2}$ \\ ${ }^{1}$ Metallurgical and Materials Engineering Department \\ ${ }^{2}$ Mechanical Engineering Department \\ Middle East Technical University, Ankara, Turkey
}

\begin{abstract}
Numerical and experimental studies have been carried out to investigate the evolution of residual stresses and microstructures in quenched steel components. In the numerical analysis, a finite element model is implemented for predicting the temperature field, phase changes with their associated internal stresses in axisymmetrical components. The model is verified by several comparisons with other known numerical results. Case studies are performed to investigate the effects of the quench bath temperature and the specimen geometry. Specimen geometry has been analyzed by introducing a hole in a cylinder and varying hole diameter and its eccentricity. Experiments include microstructural examination and $\mathrm{X}$-ray diffraction measurements of surface residual stresses.
\end{abstract}

\section{INTRODUCTION}

Quenching is a common process to produce parts with reliable service properties. Long parts with small cross sections, those with nonsymmetrical shapes, and those with holes, deep keyways or grooves are difficult to quench without distortion or cracking. Especially in quenching of construction steels, there are risks of quench cracking, and great deformation is often encountered. On the other hand, most serious residual stresses are from the differential cooling and the volume increase due to the phase transformations.

A successful hardening requires a good design, a steel with adequate hardenability, properly maintained quench facilities, the proper quenchant, and experienced technicians. Residual stress and distortion, avoidance of cracking, final microstructure and hardness distribution should be controlled to promote the desirable effects of quenching. Unfortunately, there are no state-of-the-art criteria for designing and construction of a quenching system that will provide optimal heat transfer and quench uniformity. Being able to calculate the effect of quench parameters by computer simulation would make it possible to avoid quench cracking, to control distortion and residual stress distribution, and to have optimal mechanical and metallurgical properties. 
In quench hardening of steel components, thermal stresses are produced due to large temperature gradients, and the evolution of microstructure influences the stress field substantially. Phase transformations are accompanied by volume variation and transformation plasticity. On the other hand, any stress/strain state existing in the volume affects the phase transformation behavior. During quenching, all these mechanisms cooperate, and cause a continuously changing internal stress field. When the local yield strength is exceeded at some temperature at any point, a non-uniform plastic flow occurs. This causes a total residual stress state at the end of the quench process that can be beneficial or detrimental depending on magnitude and sign of the stresses. In extreme cases the body could even crack during quenching. To predict the final dimensional size and residual stress state, the complete stress-strain history must be traced accurately.

Several studies have been conducted on the prediction of quench residual stresses. Yu calculated the residual stresses in rotationally symmetric bodies considering temperature, phase transformation and elastic-plastic deformations /1/. Hildenwall predicted residual stresses due to quenching of carburized steels $/ 2 /$. Sjoström investigated the effect of changes in radius of the cylinder, and considered the effect of stress on transformation strain as well as mixed proportions of isotropic and kinematic hardening /3/. Denis et al. concluded that transformation plasticity has a marked effect on residual stress state, and reviewed the main effects of stress on metallurgical and mechanical interactions /4/. Leblond et al. discussed the kinetics of anisothermal transformations and the influence of the transformations on transformation plasticity $15 /$. Schroder examined the effect of steel types, cylinder diameter and quenching conditions on residual stresses $/ 6 /$. Graja investigated the influence of various heat treatments on the thermal and transformation stresses, residual stresses and distortion of plain- $C$ and low-alloy steels $/ 7 /$. Inoue et al. simulated various heat treatment processes based on the thermomechanical theory $/ 8 /$.

The aim of this study is to investigate effects of the parameters of quench and specimen on the final residual stress and microstructural states by means of a finite element model. The verification of the model is carried out through comparisons with available numerical results from literature. Effects of quench bath temperature and workpiece geometry on residual stresses and microstructure are investigated numerically and experimentally. By quenching the cylinders with holes, the residual stress states and the microstructural evolution due to variation of wall-thickness is examined by $\mathrm{X}$-ray measurements and microstructural observations.

\section{FINITE ELEMENT MODEL OF QUENCHING}

The model considers thermal interactions, microstructural evolution and elasto-plastic deformation. Axisymmetrical geometry with constant axial strain is assumed, and a single strip of elements defining the $1 / 4$ of the cylinder is used in the computations. For each time step: firstly, the temperature distribution is determined. It is influenced by the quenching severity of the medium, thermal conductivity, heat capacity and latent heat due to phase transformation. Quenching severity is 
characterized by the convective heat transfer as a function of temperature at the specimen surface. Phase transformations are considered through the description of volume fractions of phases as a function of time for every cooling curve. Finally, internal stresses are computed by means of an elasto-plastic analysis assuming small strains and the Prandtl-Reuss constitutive equations. Dependence of material properties on temperature and microstructure is described by a linear mixture rule.

\section{Temperature Field}

Quenching is defined as a transient heat conduction problem with convective boundary conditions, and internal heat source and sink. The mixed boundary conditions are

$$
\begin{aligned}
& -\frac{d q(T)}{d t}=-h_{\mathcal{C}}\left(T_{s}-T_{m}\right) \\
& \left.T(r, z, t)\right|_{i=0}=T_{\text {initial }},
\end{aligned}
$$

where $\mathrm{dq} / \mathrm{dt}$ is the rate of heat flux across a boundary layer at the surface. It is dependent on the surface temperature $\left(T_{s}\right)$, temperature of the quenching medium $\left(T_{m}\right)$, and the convective heat transfer coefficient of the quenching medium $\left(h_{c}\right)$.

After standard procedures of finite element discretization, for an isotropic material and axisymmetrical geometry the elemental equation becomes

$$
[H]\{T\}+[C]\left\{\frac{d T}{d t}\right\}+\{Q\}=0
$$

The elemental thermal conductivity matrix, $[\mathrm{H}]$, includes also the convection effect for the surface nodes (at specimen/quench medium interface), and is given by

$$
\begin{aligned}
{[H]=\iiint \lambda } & {\left[\frac{\partial\{N\}}{\partial} \frac{\partial\{N\}^{T}}{\partial}+\frac{\partial\{N\}}{\partial z} \frac{\partial\{N\}^{-}}{\partial z}\right] d V } \\
& +\int j h_{h_{r}}\{N\}\{N\}^{T} d A
\end{aligned}
$$

and the elemental heat capacity matrix is formulated as

$$
[C]=r \iiint_{\rho c}\{N\}\{N\}^{T} d V
$$

The vector of external nodal heat fluxes including the internal heat generation due to phase transformation at any node and time dependent convection at surface nodes is given by

$$
\{Q\}=-\iiint_{r} r \frac{\partial L}{\partial}\{N\} d V-\iint_{h_{\mathcal{C}}}\{N\} T_{m} d A
$$


A general implicit method is used for the time marching scheme. The solution for the unknown temperature yields at time $t+\Delta t$

$$
\{\}_{t+\Delta t}-\frac{([C]-\Delta t(1-\theta)[H])\{T\}_{t}-\{\bar{Q}\} \Delta t}{[C]+\Delta t \theta[H]}
$$

Here, $\{Q\}$ represents an average value of $\{Q\}$

$$
\{\bar{Q}\}=\{Q\}_{t}+\theta\left(\{Q\}_{t+\Delta t}-\{Q\}_{t}\right)
$$

if a linear variation of $\{Q\}$ with respect to time is assumed. $\theta$ is taken as $2 / 3$ (Galerkin) scheme). The computations are terminated when the second Euclidean error norm of all nodal temperatures falls below a certain critical value in the order of $10^{-3} / 10 /$.

In the equations $\{N\}$ represents the vector of shape functions, $T$ stands for temperature, $t$ for time, $r$ for radial displacement, $\mathrm{z}$ for axial displacement, $\lambda$ for thermal conductivity, $\rho$ for density and $c$ for specific heat. $\partial L / \partial t$ is the volumetric heat source and embodies the latent heat effect of the phase transformation,

$$
\frac{\partial L}{\partial}=\rho \Delta H \frac{\partial V}{\partial}=\sum \Omega_{\mathrm{k}} \frac{d V_{k}}{d t}
$$

where $\Delta H$ is the heat released/absorbed per unit mass of steel during any phase transformation, $\partial \mathrm{V} / \partial \mathrm{t}$ is the change in the phase fraction during an infinitesimal time increment, $\Omega_{k}$ is the latent heat for each phase.

\section{Phase Transformation}

Phase transformations are modelled by discretizing the cooling curves in a succession of isothermal steps and using the IT-diagrams. The cooling curves are thought of as a series of small isothermal time steps connected by instantaneous temperature changes following constant volume fraction lines. The transformed volume fractions are then calculated isothermally during each time step. For every phase the transformation is assumed to occur between certain temperature limits.

The incubation period before decomposition of austenite to pearlite or bainite is determined by using Scheil's additivity method /11/. The growth calculations are governed by the Johnson-MehlAvrami equation. The amount of martensite is calculated by using the law established by Koistinen and Marburger /13/.

\section{Stress State}

Stresses are expressed in terms of strain rates. The total engineering strain rate tensor is defined as 
the sum of several parts. The elastic strain tensor is given by

$$
\varepsilon_{i j}^{e}=\frac{1}{E}\left[(1+v) \sigma_{i j}-\delta_{i j} v \sigma_{h}\right]
$$

where $E$ is the Young's Modulus and $v$ the Poisson's ratio, $\sigma_{i j}$ the nominal stress tensor (approximately equal to the Cauchy stress tensor for small strains), $\sigma_{\mathrm{h}}$ is the hydrostatic stress and $\delta_{\mathrm{ij}}$ is the Kronecker's delta. The thermal strain is formulated by

$$
\varepsilon_{i j}^{i h}=\sum_{k=1}^{p} V_{k} \int_{0}^{T} \alpha_{k} d T
$$

where $\alpha_{k}$ is the thermal expansion coefficient of phase $k$ and $p$ is the total number of phases. The transformation plasticity is not considered. The transformation strain, $\varepsilon_{k}{ }^{r}$ associated with the transformation from austenite into the phase $\mathrm{k}$, is obtained by (it is assumed to be zero for austenite at $\left.0^{\circ} \mathrm{C}\right)$

$$
\varepsilon_{i j}^{t r}=\sum_{k=1}^{p} V_{k} \varepsilon_{k}^{t r}
$$

A functional is defined to compute the plastic strain rates,

$$
\phi=F\left(\sigma_{i j}\right)-\left(\sigma_{f}\left(T, V_{k}, \varepsilon^{p}\right)\right)^{2}
$$

where $\varepsilon^{p}$ is effective plastic strain, $F\left(\sigma_{i j}\right)$ is the plastic potential and it is a scalar function of deviatoric stress tensor $\mathrm{S}_{\mathrm{ij}}$

$$
F\left(\sigma_{i j}\right)=\frac{3}{2} S_{i j} S_{i j}
$$

For plastic deformation to occur, the stresses must remain on the yield surface. The Prandtl-Reuss flow rule characterizes the plastic flow duning quenching,

$$
\frac{d \varepsilon_{i j}^{p}}{d t}=d \lambda \frac{\partial \phi}{\partial \sigma_{i j}}=d \lambda \frac{\partial F}{\partial S_{i j}}
$$

where $d \lambda$ is the plastic multiplier. The equivalent plastic strain rate is determined by

$$
\frac{d \varepsilon^{p}}{d t}=\sqrt{\frac{2}{3} \frac{d \varepsilon_{i j}^{p}}{d t} \frac{d \varepsilon_{i j}^{p}}{d t}}
$$

Since the memory of plastic deformation history is stored by dislocation pile-ups and entanglements, the plastic strains in the austenite will be lost totally or partially during phase 
transformations $/ 3 /$. Therefore a new parameter, $\kappa$, is used in the yield function (13) instead of effective plastic strain to determine the amount of actual strain hardening in any phase $k$.

$$
\phi=F\left(\sigma_{i j}\right)-\left(\sigma_{f}\left(T, V_{k}, K_{k}\right)\right)^{\alpha}
$$

Then, to enforce the consistency condition it is necessary to define the yield surface and hence to determine the hardening parameter at the end of the time step. Assuming isotropic linear strain hardening, a variable flow stress is defined

$$
\sigma_{\mathrm{f}}=\mathrm{Y}+\mathrm{H} \varepsilon^{\mathrm{p}}
$$

where $\mathrm{Y}$ is the instantaneous yield stress, and $\mathrm{H}$ is the incremental-secant modulus.

The specimen is subjected to initial strain increments due to temperature change and phase transformation. Stress increments are caused by the difference between the increments of actual and initial strains,

$$
\frac{d \sigma_{i j}}{d t}=\left[D_{e}\right\}\left(\frac{d \varepsilon_{i j}}{d t}-\frac{d \varepsilon_{i j}^{t h}}{d t}-\frac{d \varepsilon_{i j}^{t r}}{d t}\right)
$$

where $\left[D_{0}\right]$ is the elastic constitutive matrix defined as

$$
\left[D_{e}\right]=\frac{E}{(1+v)(1-2 v)}\left[\begin{array}{ccc}
1-v & v & v \\
v & 1-v & v \\
v & v & 1-v
\end{array}\right]
$$

The plastic strain increment will occur only if the elastic stress increment puts the stress state outside the yield surface. Then, the elastic-plastic matrix $\left[\mathrm{D}_{\text {op }}\right]$ (standard tangent modulus) takes the place of $\left[D_{e}\right]$ for the elastic-plastic part of the analysis,

$$
\left.\left[D_{e p}\right]=D_{e}\right]\left(1-\frac{\frac{\partial F_{i}}{\partial \sigma_{i}}\left[D_{e}\right]^{T} \frac{\partial F^{T}}{\partial \sigma_{y}}}{A^{\prime}+\frac{\partial F}{\partial \sigma_{i j}}\left[D_{l}\right]^{T} \frac{\partial F}{\partial \sigma_{i j}}}\right)
$$

where $A^{\prime}$ is a constant dependent on yield stress, strain hardening coefficient, volume fraction of phases, plastic strains and plastic potential /10/. 


\section{Solution Algorithm}

The load history is divided into incremental load steps. At each step, after computing the elemental mean values of temperatures and volume fraction of phases, nodal displacements are determined from the global stiffness equations as obtained by assembling the element stiffness equations. The strain and stress components are then computed at the center of each element. If the updated stress state is within the yield surface, the material within the respective element is assumed to have either remained elastic or to have unloaded elastically. However, if the elastic stress state is outside the yield surface, an integration procedure (a generalized trapezoidal rule /12/) is adopted (Figure 1),

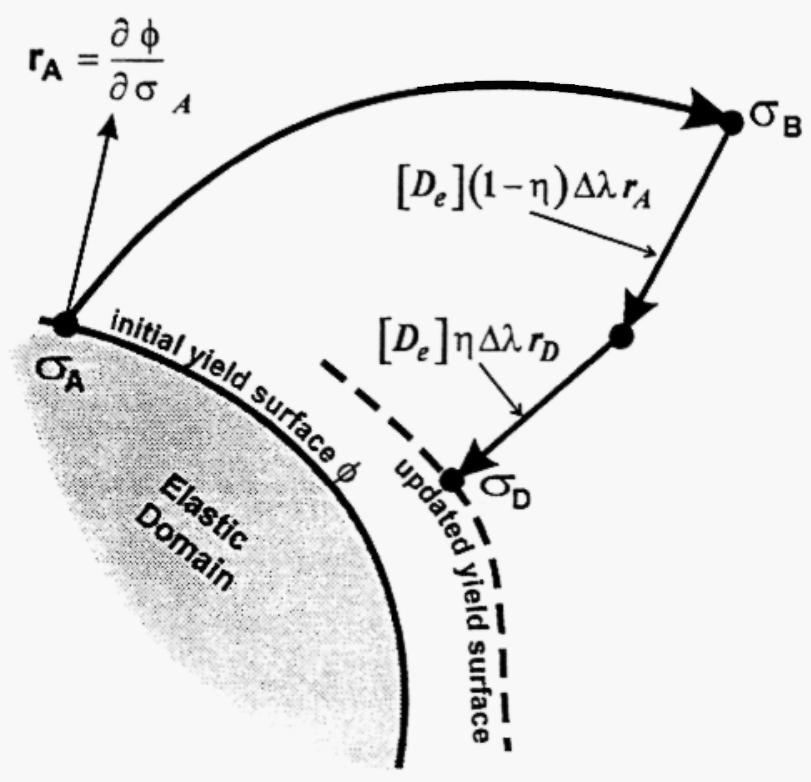

Fig. 1: Schematic representation of the return mapping method

$$
\begin{aligned}
& \sigma_{D}=\sigma_{A}+\left[D_{e}\right]\left(\Delta \varepsilon-\Delta \varepsilon^{p}\right)=\sigma_{B}-\left[D_{e}\right] \Delta \varepsilon^{p} \\
& \Delta \varepsilon^{p}=\Delta \lambda\left[\left.(1-\eta) \frac{\partial \phi}{\partial \sigma}\right|_{A}+\left.\eta \cdot \frac{\partial \phi}{\partial \sigma}\right|_{D}\right.
\end{aligned}
$$

where $A$ is the starting stress state, $B$ the estimated end state not satisfying the yield condition, and $D$ is the corrected stress state on the yield surface. In the computations, a tangent stiffness-radial corrector method is used, i.e., assuming von Mises yield condition, associated flow rule and isotropic strain hardening, and $\eta$ is zero. 
Iteration is terminated when the ratio of the second Euclidean norms of the difference of the nodal force and the force vector falls below a critical value $\left(10^{-3}\right)$. The ratio of equivalent stress to yield stress is used as an indication of the efficiency for the method to bring the equivalent stress back to the yield surface. If it is near 1 , this means that the technique is bringing the plastic stresses back to the yield surface.

\section{NUMERICAL VERIFICATION; QUENCHING OF Ck45 STEEL}

A Ck45 steel cylinder of $30 \mathrm{~mm}$ diameter is quenched from $680^{\circ} \mathrm{C}$ into $20^{\circ} \mathrm{C}$ oil. A constant convective heat transfer coefficient of $3000 \mathrm{~W} / \mathrm{m}^{2 \circ} \mathrm{C}$ is used. The physical and mechanical data is in Table 1. Our own numerical results are compared with experimental neutron diffraction and also numerical results as given by Schröder /6/. Our own computations were conducted with 15 isoparametric quadrilateral ring elements and a time step of 0.001 seconds.

Table 1

Physical and mechanical data

\begin{tabular}{|c|c|c|c|c|c|c|c|}
\hline $\begin{array}{c}\mathbf{T} \\
\left({ }^{\mathbf{0}} \mathbf{C}\right)\end{array}$ & $\begin{array}{c}\mathbf{E} \\
(\mathbf{G P a})\end{array}$ & $U$ & $\begin{array}{c}\mathbf{Y} \\
(\mathbf{M P a})\end{array}$ & $\begin{array}{c}\mathbf{H} \\
(\mathbf{G P a})\end{array}$ & $\begin{array}{c}\alpha \\
\left(\mathbf{1}^{\circ} \mathbf{C}\right)\end{array}$ & $\begin{array}{c}\lambda \\
\left(\mathbf{J} / \mathbf{~ m s}{ }^{{ }^{\circ}} \mathbf{C}\right)\end{array}$ & $\begin{array}{c}\mathbf{C} \rho \\
\left(\mathbf{M J} / \mathbf{m}^{\mathbf{~}} \mathbf{C}\right)\end{array}$ \\
\hline 0 & 210 & 0.28 & 360 & 0 & $1410^{-6}$ & 49.0 & 3.78 \\
\hline 300 & 193 & 0.30 & 230 & 0 & $1410^{-6}$ & 41.7 & 4.46 \\
\hline 600 & 165 & 0.31 & 140 & 0 & $1410^{-6}$ & 34.3 & 5.09 \\
\hline 900 & 120 & 0.33 & 30 & 0 & $1410^{-6}$ & 27.0 & 5.74 \\
\hline
\end{tabular}

Figure 2 shows the comparison of our numerical results of the axial residual stresses with the neutron diffraction measurements and numerical results given by Schröder $/ 6 /$. They are in agreement with each other. Like the axial residual stresses, the tangential residual stresses are tensile in the center and compressive in the surface. The variations of effective stress and yield stress values with respect to each other are illustrated in Figure 3 for the core and the surface.

The complete history of effective and yield stresses gives information about the plastic deformation during quenching and therefore dictates the final residual stress state. Figure 3 shows that the plastic deformation occurs at the outer surface first, and then in the core. The core deforms plastically for a very short time while more plastic deformation occurs and continues to a greater extent at the surface. The elements are subjected to plastic deformation due to low yield strengths at higher temperatures. However, since the surface cools faster than the core, the yield stress increases at a faster rate and allows the outer sections of the cylinder to unload elastically. After elastic unloading both in the core and at the surface, the effective stresses decrease suddenly to very low values. Then they increase to values of up to $100 \mathrm{MPa}$ in the center and 280 at the surface. 


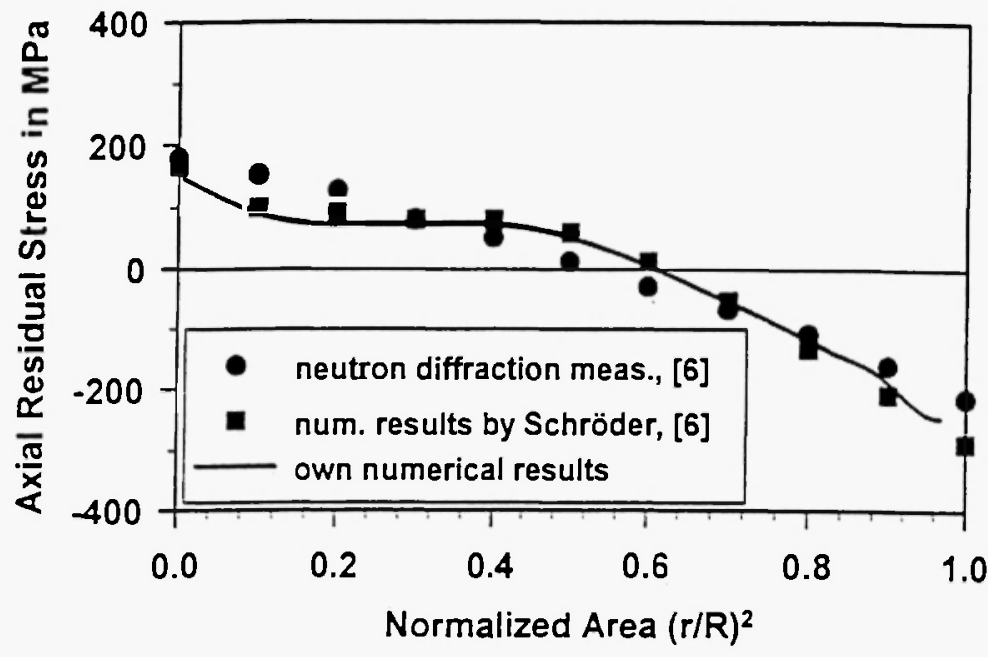

Fig. 2: Comparison of numerically predicted axial residual stress (RS) with results given by Schröder $16 /$
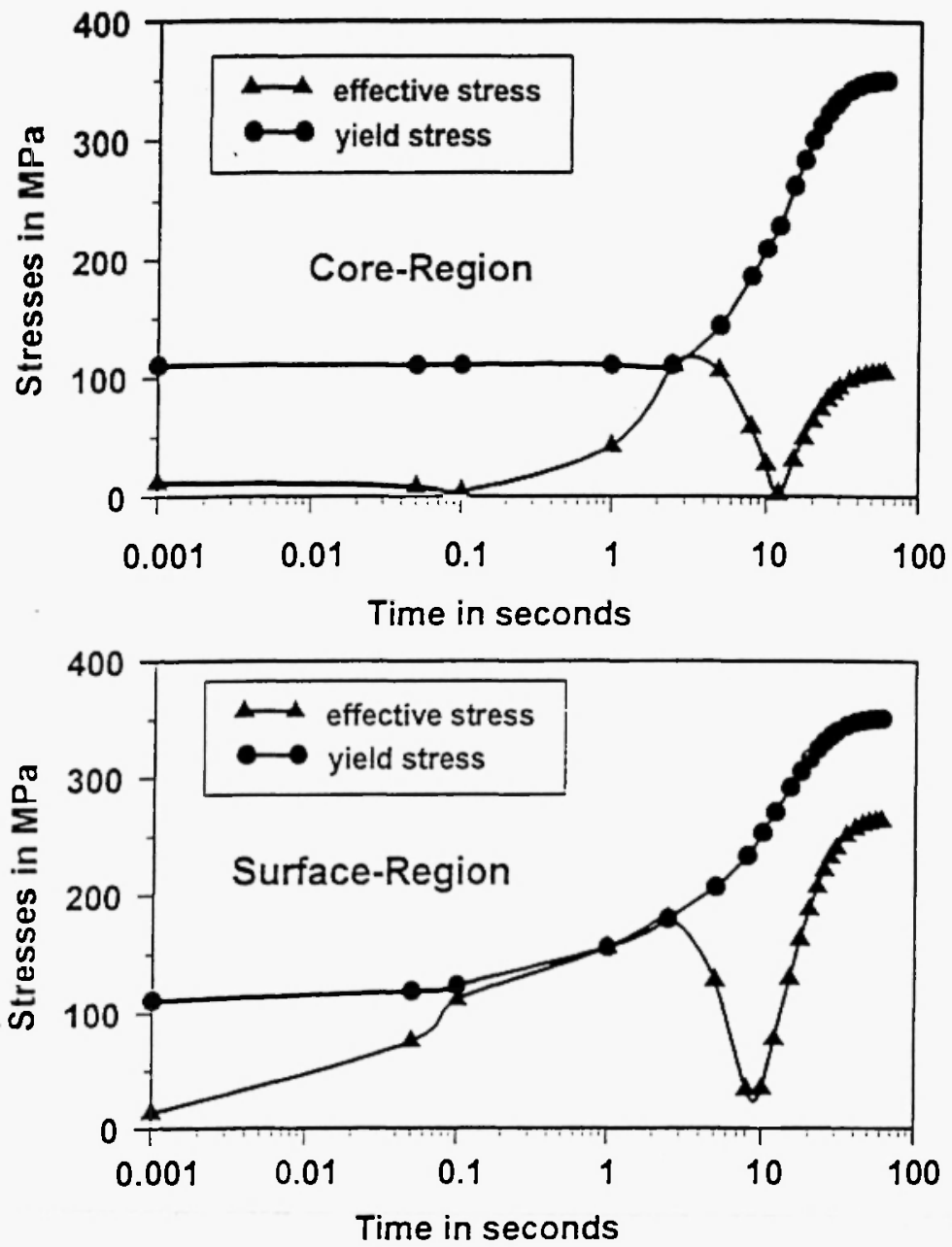

Fig. 3: Variation of yield stress and effective internal stress values for the center and the surface during quenching of a $\mathrm{Ck} 45$ steel cylinder from $680^{\circ} \mathrm{C}$ into $20^{\circ} \mathrm{C}$ water. 


\section{EFFECT OF QUENCH BATH TEMPERATURE ON QUENCHING OF C60 STEEL}

\section{Quenching without phase transformation}

C60 steel cylinders of $30 \mathrm{~mm}$ diameter were quenched from $720^{\circ} \mathrm{C}$ to $20^{\circ} \mathrm{C}$, and to $60^{\circ} \mathrm{C} / 6 /$. Temperature dependent mechanical and physical properties are given in Tables 2 and 3. Table 4 gives the temperature dependence of convective heat transfer coefficients. The finite element mesh consists of 15 elements and 32 nodes. Time increment size equals 0.002 seconds.

Quenching into $20^{\circ} \mathrm{C}$ water results in a faster cooling rate with respect to $60^{\circ} \mathrm{C}$ water quenching both in the core and on the surface; however, the surface is more affected (Figure 4). The thermal gradients in the outer region of the cylinder are substantially reduced in the $60^{\circ} \mathrm{C}$ water quench Since temperature differences between the surface and the center are higher in the case of $20^{\circ} \mathrm{C}$ water quench, there are higher thermal stresses during quench, and therefore, the amount of plastic strains increase. The specimen has a residual stress distribution that is similar to the $60^{\circ} \mathrm{C}$ water quench, but higher in magnitude. Since there is less plastic deformation in the $60^{\circ} \mathrm{C}$ water quench the magnitude of compressive and tensile stresses is reduced when compared to the $20^{\circ} \mathrm{C}$ water quench.

Figure 5 illustrates the axial and the effective residual stresses. The specimen quenched into $20^{\circ} \mathrm{C}$ water has higher residual stresses than the one quenched into $60^{\circ} \mathrm{C}$ water. There are compressive residual stresses in the surface and tensile residual stresses in the core. The magnitude of the tensile residual stresses in the core reaches considerably high values of above $600 \mathrm{MPa}$. The possibility of

Table 2

Heat transfer data for $\mathrm{C} 60$ steel

\begin{tabular}{|c|c|c|}
\hline $\mathrm{T}\left({ }^{\circ} \mathbf{C}\right)$ & $\lambda\left[\mathrm{J} / \mathbf{m s}^{\mathbf{0}} \mathbf{C}\right]$ & $\mathbf{C} \rho\left[\mathbf{M J} / \mathbf{m}^{30} \mathbf{C}\right]$ \\
\hline 0 & 15.0 & 4.15 \\
\hline 300 & 18.0 & 4.40 \\
\hline 600 & 21.7 & 4.67 \\
\hline 900 & 25.1 & 4.90 \\
\hline
\end{tabular}

Ferrite, Pearlite and Bainite

\begin{tabular}{|c|c|c|}
\hline $\mathrm{T}\left({ }^{0} \mathrm{C}\right)$ & $\lambda\left[\mathrm{J} / \mathrm{ms}^{\mathbf{0}} \mathrm{C}\right]$ & $\mathrm{C} \rho\left[\mathrm{MJ} / \mathrm{m}^{30} \mathrm{C}\right]$ \\
\hline 0 & 49.0 & 3.78 \\
\hline 300 & 41.7 & 4.46 \\
\hline 600 & 34.3 & 5.09 \\
\hline 900 & 27.0 & 5.74 \\
\hline \multicolumn{3}{|c|}{ Martensite } \\
\hline $\mathbf{T}\left({ }^{\circ} \mathrm{C}\right)$ & $\lambda\left[\mathrm{J} / \mathrm{ms}^{0} \mathrm{C}\right]$ & $\mathrm{C} \rho\left[\mathrm{MJ} / \mathrm{m}^{30} \mathrm{C}\right]$ \\
\hline 0 & 43.1 & 3.76 \\
\hline 300 & 36.7 & 4.45 \\
\hline 600 & 30.1 & 5.07 \\
\hline
\end{tabular}


Table 3

Mechanical data for C60 steel /1/

Austenite

\begin{tabular}{|c|c|c|c|c|c|}
\hline $\begin{array}{c}\mathbf{T} \\
\left({ }^{\mathbf{0}} \mathbf{C}\right)\end{array}$ & $\begin{array}{c}\mathbf{E} \\
(\mathbf{G P a})\end{array}$ & $V$ & $\begin{array}{c}\sigma_{\mathbf{y}} \\
(\mathbf{M P a})\end{array}$ & $\begin{array}{c}\mathbf{H} \\
(\mathbf{G P a})\end{array}$ & $\begin{array}{c}\alpha \\
\left(\mathbf{1}^{\mathbf{0}} \mathbf{C}\right)\end{array}$ \\
\hline 0 & 200 & 0.29 & 220 & 1000 & $\begin{array}{c}21.7 \\
10^{-6}\end{array}$ \\
\hline 300 & 175 & 0.31 & 130 & 16000 & 21.7 \\
& & & & & $10^{-6}$ \\
\hline 600 & 150 & 0.33 & 35 & 10000 & 21.7 \\
& & & & & $10^{-6}$ \\
\hline 900 & 124 & 0.35 & 35 & 500 & 21.7 \\
& & & & & $10^{-6}$ \\
\hline
\end{tabular}

Ferrite, Pearlite,Bainite

\begin{tabular}{|c|c|c|c|c|c|}
\hline $\mathbf{T}\left(^{\mathbf{0}} \mathbf{C}\right)$ & $\begin{array}{c}\mathbf{E} \\
(\mathbf{G P a})\end{array}$ & $v$ & $\begin{array}{c}\sigma_{\mathbf{y}} \\
(\mathbf{M P a})\end{array}$ & $\begin{array}{c}\mathbf{H} \\
(\mathbf{G P a})\end{array}$ & $\begin{array}{c}\alpha \\
(\mathbf{1 / 0} \mathbf{C})\end{array}$ \\
\hline 0 & 210 & 0.28 & 450 & 1000 & $\begin{array}{c}15.3 \\
10^{-6}\end{array}$ \\
\hline 300 & 193 & 0.30 & 230 & 16000 & $\begin{array}{c}15.3 \\
10^{-6}\end{array}$ \\
\hline 600 & 165 & 0.31 & 140 & 10000 & $\begin{array}{c}15.3 \\
10^{-6}\end{array}$ \\
\hline 900 & 120 & 0.33 & 30 & 500 & $\begin{array}{c}15.3 \\
10^{-6}\end{array}$ \\
\hline
\end{tabular}

Martensite

\begin{tabular}{|c|c|c|c|c|c|}
\hline $\mathrm{T}\left({ }^{0} \mathrm{C}\right)$ & $\begin{array}{c}\mathbf{E} \\
(\mathbf{G P a})\end{array}$ & $v$ & $\begin{array}{c}\sigma_{\mathbf{y}} \\
(\mathbf{M P a})\end{array}$ & $\begin{array}{c}\mathbf{H} \\
(\mathbf{G P a})\end{array}$ & $\begin{array}{c}\alpha \\
\left(1 /{ }^{0} \mathrm{C}\right)\end{array}$ \\
\hline 0 & 200 & 0.28 & 1750 & 1000 & $1310^{-6}$ \\
\hline 300 & 185 & 0.30 & 1550 & 16000 & $13 \quad 10^{-6}$ \\
\hline 600 & 168 & 0.31 & 1350 & 10000 & $1310^{-6}$ \\
\hline
\end{tabular}

Table 4

Convective heat transfer coefficients for $20^{\circ} \mathrm{C}$ and $60^{\circ} \mathrm{C}$ water quench /4/

\begin{tabular}{|c|c|c|c|}
\hline $20^{\circ} \mathrm{C}$ & & $60^{\circ} \mathrm{C}$ & \\
\hline$T\left({ }^{0} \mathrm{C}\right)$ & $h_{c}\left|J / m^{2} s^{0} C\right|$ & $\mathrm{T}\left({ }^{0} \mathrm{C}\right)$ & $h_{c}\left[J / m^{2} s^{0} C\right]$ \\
\hline 0 & 4350.0 & 0 & 135.3 \\
\hline 200 & 8207.1 & 200 & 2029.2 \\
\hline 400 & 11961.7 & 400 & 2840.9 \\
\hline 430 & 13491.7 & 445 & 3291.2 \\
\hline 500 & 12500.0 & 500 & 3422.0 \\
\hline 560 & 10206.2 & 570 & 2610.9 \\
\hline 600 & 7793.0 & 600 & 2157.0 \\
\hline 700 & 2507.0 & 800 & 230.3 \\
\hline 800 & 437.1 & 900 & 135.2 \\
\hline 900 & 135.3 & - & - \\
\hline
\end{tabular}




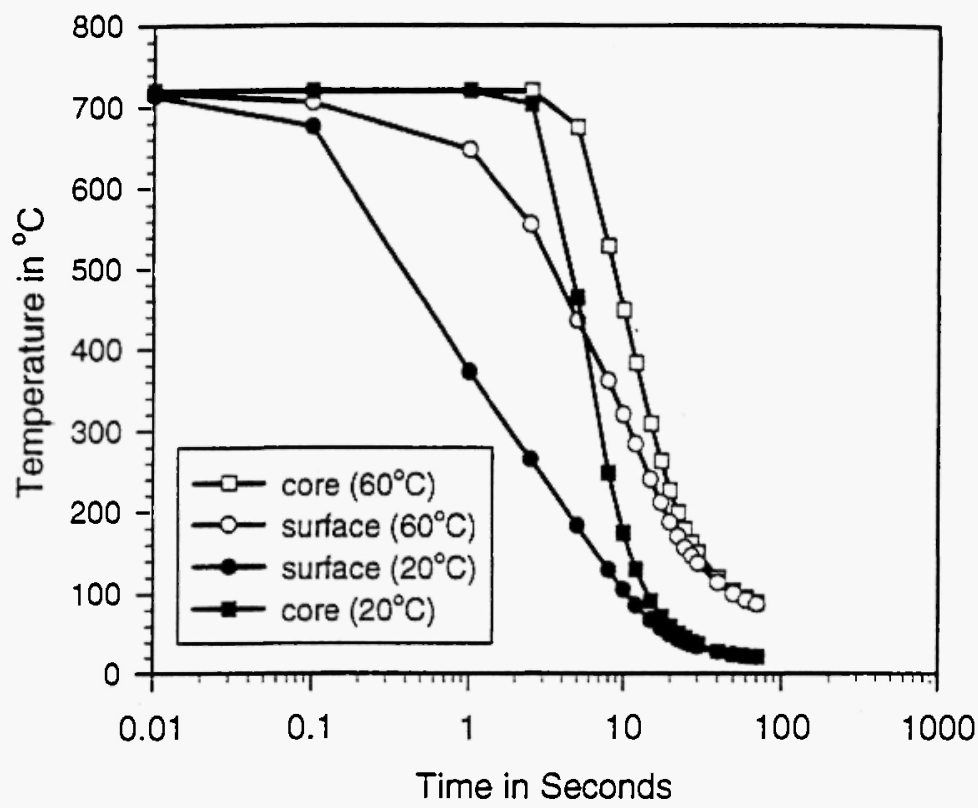

Fig. 4: Cooling curves of $20^{\circ} \mathrm{C}$ and $60^{\circ} \mathrm{C}$ water quenching from $720248 \mathrm{C}$ for $\mathrm{C} 60$ steel cylinder of 30 mm diameter.

quench cracking is relatively high in this case. The distribution of tangential residual stress is similar to that of axial residual stresses. However, the magnitude of tensile stress in the center is lower and that of compressive stress is higher at the surface.

\section{Quenching with phase transformation}

C60 steel cylinders of $30 \mathrm{~mm}$ diameter were quenched this time from the austenitization temperature of $830^{\circ} \mathrm{C}$ into $60^{\circ} \mathrm{C}$ water, and into $20^{\circ} \mathrm{C}$ water. The values given in Tables $2-4$ are used as input data. The finite element mesh consists of 10 elements and 22 nodal points. Time increment is 0.002 seconds.

During quenching, plastic deformation is caused by temperature gradients or phases change or a combination of both factors. The specimen quenched into $20^{\circ} \mathrm{C}$ water cools faster than one quenched into $60^{\circ} \mathrm{C}$ water. The differences between the convective heat transfer coefficients are very high as seen in Table 4. Since the temperature gradient is high, thermal stresses are higher. Besides, the microstructural evolution is different.

To understand the reason for the residual stress state, it is necessary to trace the stress-strain history together with the microstructural evolution. For both cases, the amount of phases along the radius is given in Figure 6, and the distributions of axial and tangential residual stresses are shown in Figure 7.

In $60^{\circ} \mathrm{C}$ water quenching, austenite almost completely transforms into pearlite and bainite in the 

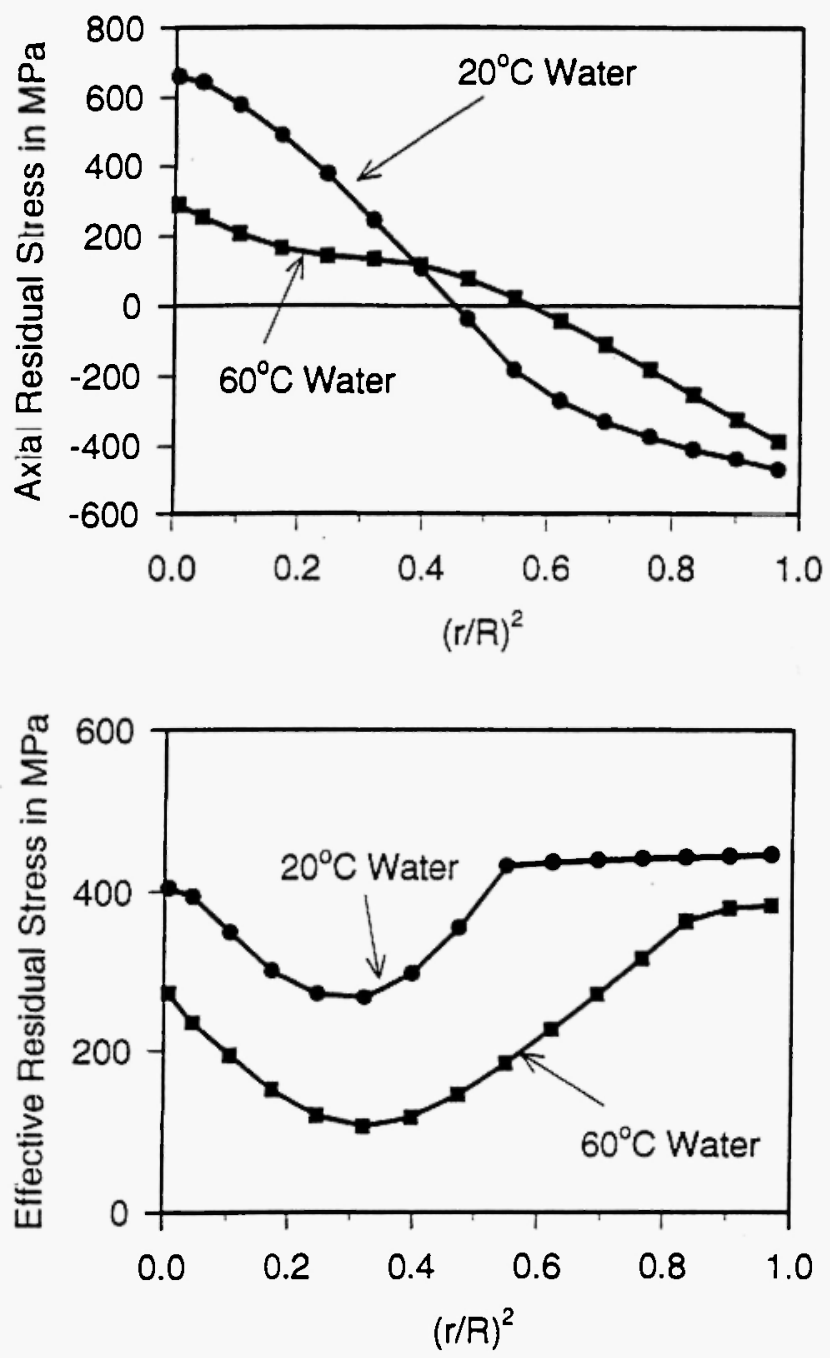

Fig. 5: Axial and effective residual stresses in the $\mathrm{C} 60$ steel cylinders, quenched from $720^{\circ} \mathrm{C}$ into $20^{\circ} \mathrm{C}$ and $60^{\circ} \mathrm{C}$ water.

entire volume. In $20^{\circ} \mathrm{C}$ water quenching, the regions near to the surface transform completely to martensite while the core transforms to pearlite, bainite and partially to martensite. During the first stage of $20^{\circ} \mathrm{C}$ water quenching, the stresses are due only to the temperature gradient, and the surface is in tension while the center is in compression. The surface has an austenitic structure; therefore, if the stress components are high enough, it yields rather than cracks. During the second stage, the surface transforms to martensite while the core transforms to pearlite or bainite. The entire piece is expanding, but since the expansion due to the formation of martensite is greater than that resulting from the formation of pearlite, the surface tends to expand more than the center. This puts the center in tension while the surface is in compression. 


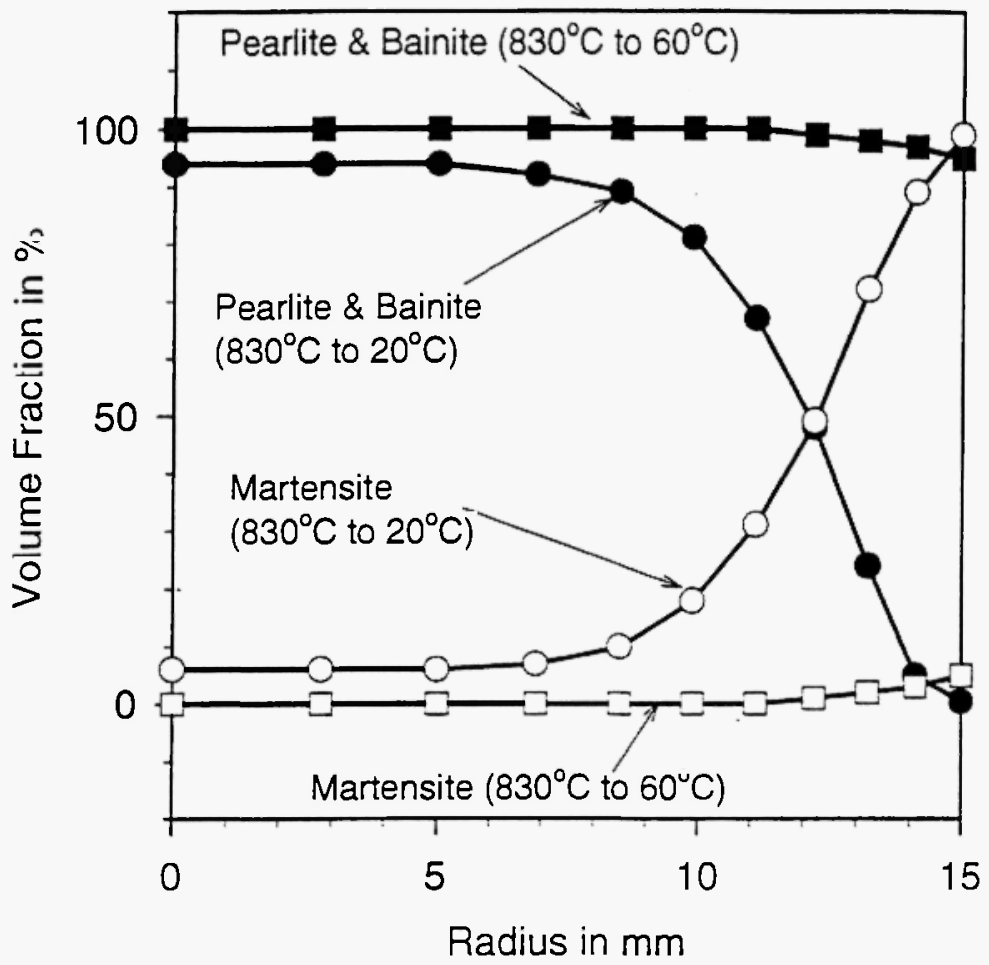

Fig. 6: Phase compositions along the radius in the $\mathrm{C} 60$ steel cylinders, quenched from $830^{\circ} \mathrm{C}$ into $20^{\circ} \mathrm{C}$ and $60^{\circ} \mathrm{C}$ water.

However, since both the surface and center are expanding, the tensile stress is small. In the third stage, after the transformations in the core and surface are finished, the center contracts on cooling from the transformation temperature to room temperature. The surface, being martensitic and having reached room temperature much earlier, prevents the center from contracting as much as it should. This results in higher tensile stresses in the center. Since the center is pearlite of relatively low tensile strength, the greatest danger of cracking exists during this stage.

\section{EFFECT OF WORKPIECE GEOMETRY ON QUENCHING OF C60 AND 90MnCrV8 STEELS}

Temperature gradients are largely a function of the size and shape of the component. Therefore, a good design requires planning of such shapes that will have minimum temperature gradients throughout the component during quenching. Drilled cylinders may provide a reference basis to investigate the quantitative interdependencies among shape and size of the component, heat treatment procedures and process parameters, mechanical and metallurgical properties. By changing the diameter 

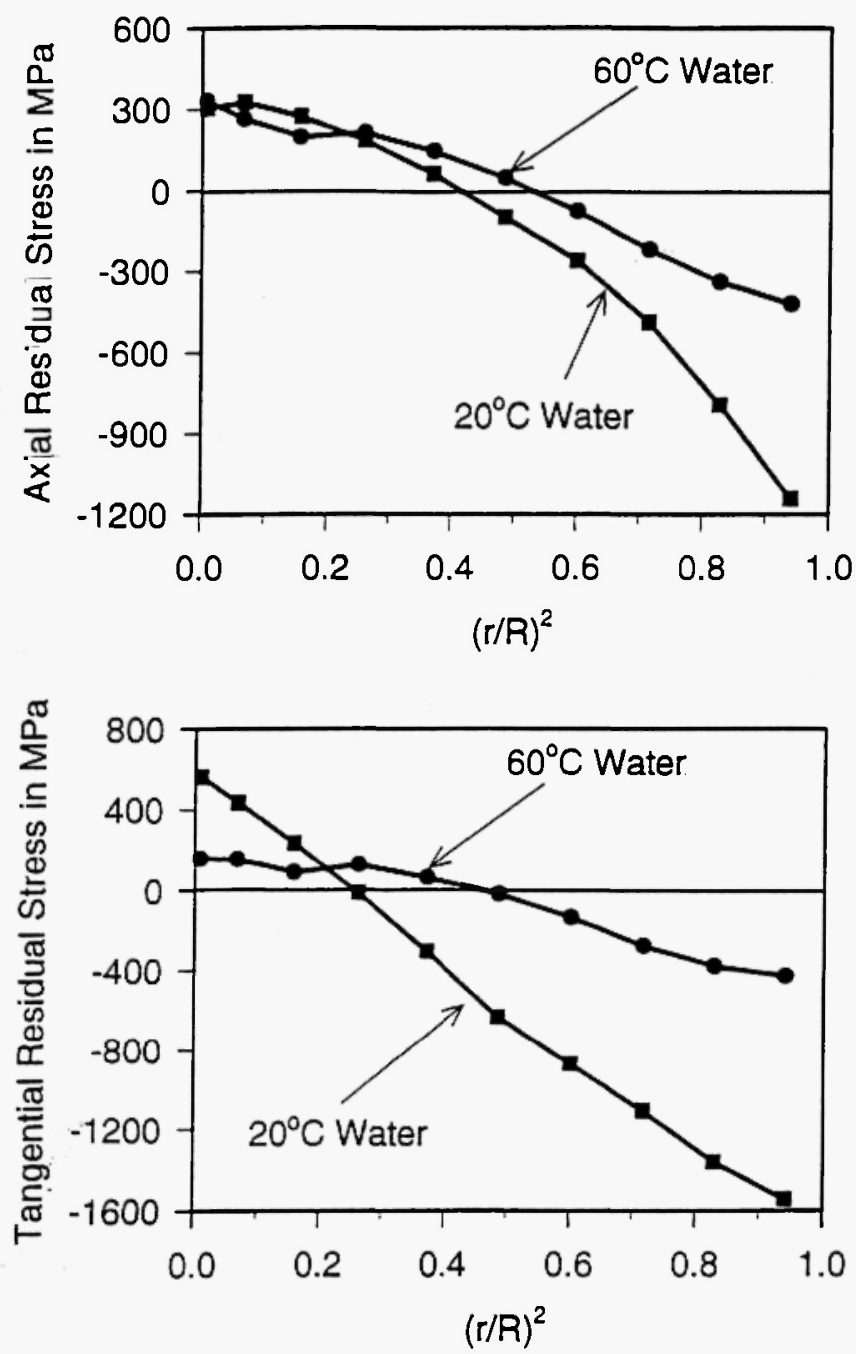

Fig. 7: Distribution of residual stresses in the $\mathrm{C} 60$ steel cylinder of 30 nun diameter, quenched from $830^{\circ} \mathrm{C}$ into $20^{\circ} \mathrm{C}$ and $60^{\circ} \mathrm{C}$ water.

and the position of the hole in the specimen, various variations in wall thickness can be obtained.

Specimens have been prepared as centrically or eccentrically drilled cylinders (Figure 8). The cylinders are of $\mathrm{C} 60$ and $90 \mathrm{MnCrV8}$ steels, and have diameters of $30 \mathrm{~mm}$. Their detailed identifications are in Table 5. Specimens are quenched from $830^{\circ} \mathrm{C}$ into $60^{\circ} \mathrm{C}$ water, while $90 \mathrm{MnCrV8}$ steel specimens are oil-quenched from $820^{\circ} \mathrm{C}$. All are tempered at $200^{\circ} \mathrm{C}$ for 1.5 hours. To protect the holes against the quenching effect, both ends of the drilled cylinders are closed with steel blocks.

Surface residual stresses have been measured using a stationary $\Psi$-type X-ray diffractometer. The $\mathrm{d}-\sin ^{2} \Psi$ method has been used to calculate residual stresses. All measurements have been carried out at the middle height of the specimens. In case of eccentrically drilled cylinders the residual stresses have 


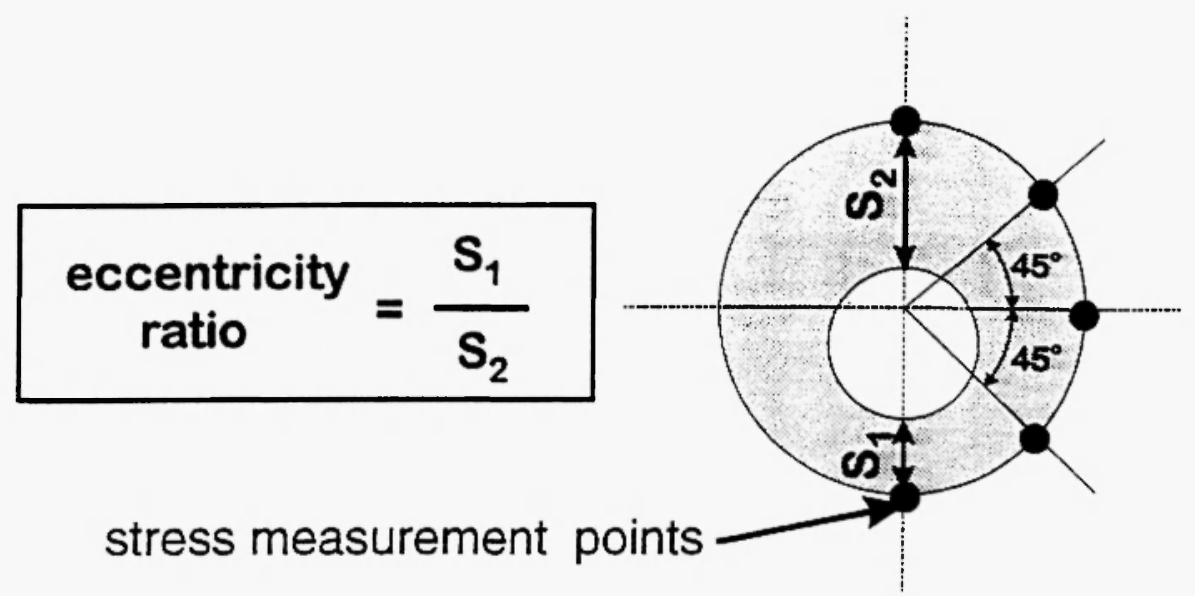

Fig. 8: Geometry of an eccentrically drilled cylinder and X-ray measurement points.

Table 5

Identification of specimens (C60 steel: $0.6 \% \mathrm{C}, 0.25 \% \mathrm{Si}, 0.75 \% \mathrm{Mn}$, 90MnCrV8: $0.9 \% \mathrm{C}, 2 \% \mathrm{Mn}, 0.35 \% \mathrm{Cr}, 0.2 \% \mathrm{~V}$ )

\begin{tabular}{|c|c|c|c|c|}
\hline C60 & 90 Mn CrV8 & Hole dia. $[\mathbf{m m} \mid$ & $\begin{array}{c}\text { Hole dia. } / \\
\text { outer dia. }\end{array}$ & $\begin{array}{c}\text { Eccentricity ratio } \\
\mathbf{S}_{\mathbf{1}} / \mathbf{S}_{\mathbf{2}}\end{array}$ \\
\hline A2 & F2 & & & 1.00 \\
A3 & F3 & 6.0 & 0.2 & 0.50 \\
A4 & F4 & & & 0.25 \\
\hline B2 & G2 & & 0.3 & 1.00 \\
B3 & G3 & 9.0 & & 0.50 \\
B4 & G4 & & & 0.25 \\
\hline C2 & H2 & & 0.4 & 1.00 \\
C3 & H3 & 12.0 & & 0.50 \\
C4 & H4 & & & 0.25 \\
\hline D2 & I2 & & & 1.00 \\
D3 & I3 & 15.0 & & 0.50 \\
D4 & I4 & & & 0.25 \\
\hline E2 & J2 & & & 1.00 \\
E3 & J3 & 18.0 & & 0.50 \\
E4 & J4 & & & 0.25 \\
\hline
\end{tabular}

been measured at certain points along the circumference (Figure 8). For hollow cylinders, several measurements have been carried out along the circumference of the cylinders, and then an average of these values has been taken. In the measurements, deviations up to $40 \mathrm{MPa}$ have been observed.

Residual stress measurements, given in Figure 9, indicate that C60 steel is very sensitive to the variation in wall thickness. In the series of hollow cylinders (A2-E2), starting from B2 a transition from 
increasing eccentricity

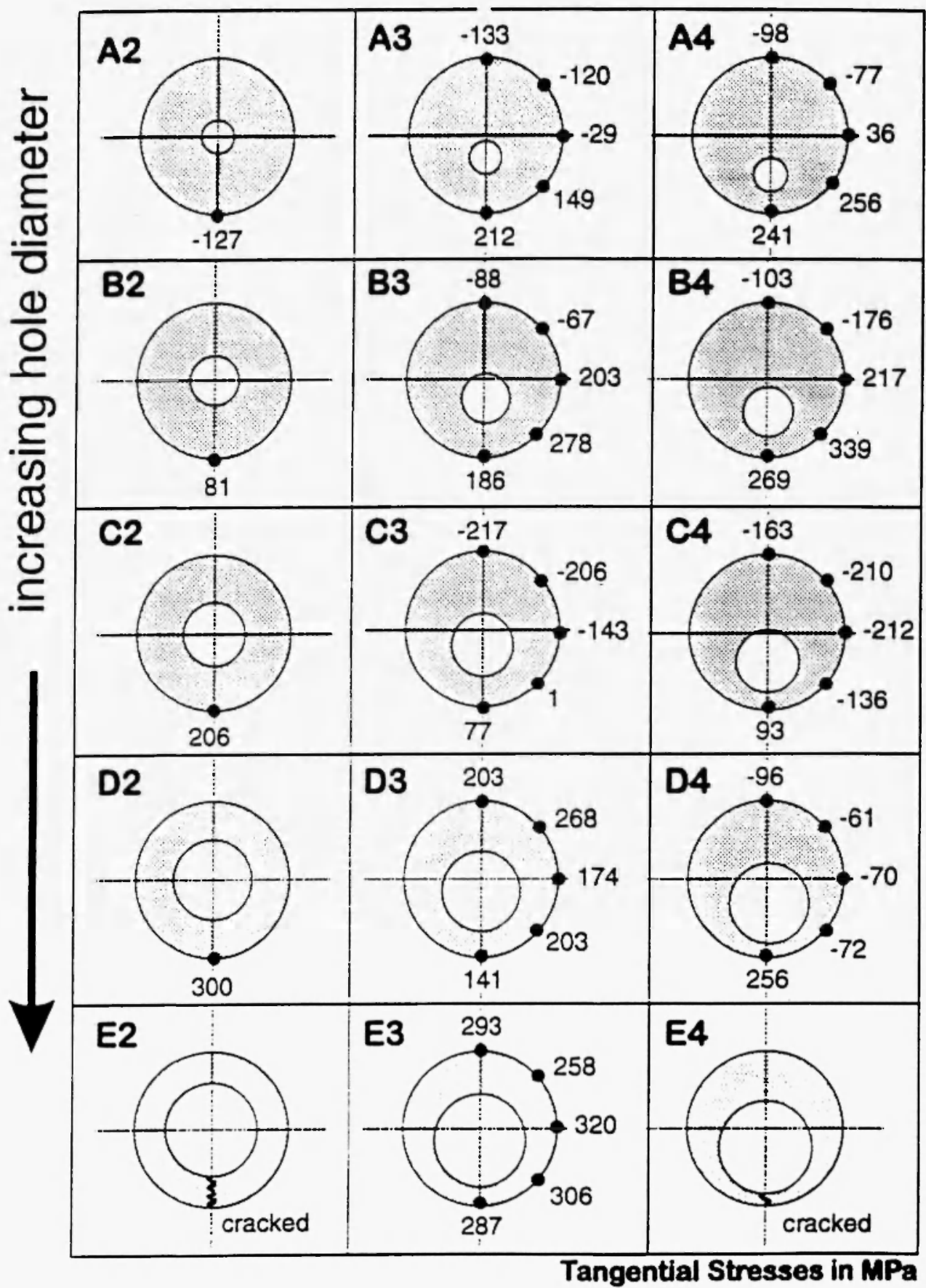

Fig. 9: Tangential residual stresses along the circumference of the $\mathrm{C} 60$ steel cylinders of $30 \mathrm{~mm}$ in diameter, measured by $\mathrm{x}$-ray diffraction. 
compressive to tensile residual stresses is observed. As the hole diameter increases the amount of residual stresses increases; moreover, quench cracking occurs in the cylinder with $18 \mathrm{~mm}$ diameter hole. In eccentrically drilled cylinders, there exists a transition zone from compressive to tensile residual stresses along the circumference, except D3 and E3, whose surfaces are completely under the effect of high tensile residual stresses. In the A3-E3 series, the residual stresses show a noticeable variation along the circumference. In the specimen $\mathrm{E3}$, the values along the circumference become almost uniform When the hole diameter increases, the effect of eccentricity decreases in this specimen series. In the A4-E4 series, the points of the thinnest wall thickness are more critical for cracking. They have higher tensile residual stresses at these points, and the specimen $\mathrm{E} 4$ was cracked at its thinnest point. It is clear that in the case of $\mathrm{C} 60$ steel the diameter and eccentricity degree of the hole cause a complex state of residual stresses. In the series A2-A4 and B2-B4 there are drastic variations in the tangential residual stresses in the range between -127 and $339 \mathrm{MPa}$. However, the effect of eccentricity on the residual stresses decreases starting from the $\mathrm{D}$ series.

The variation of wall thickness affects not only the thermal gradients but also the microstructural evolution. As the wall thickness decreases the cooling rate in the whole cylinder increases, and transformations start earlier. The amount of martensite in the surface increases as the hole diameter increases, and martensitic transformation occurs also in the core. In the cylinder with $18 \mathrm{~mm}$ hole, it reaches very high amounts in the core while the surface has a completely martensitic structure. In other words, a transition occurs to the through hardening case (i.e., the whole cylinder is fully martensitic). Therefore, the residual stress state is completely reversed, i.e., tension in the surface and compression in the core.

$90 \mathrm{MnCrV8}$ steel cylinders are not sensitive to the variation in the wall-thickness under the applied quenching procedure. The amount of retained austenite at the surface is about $16 \%$. All cylinders have tensile residual stresses at their surfaces. Wall-thickness variations do not show any considerable effect on the surface residual stresses. Independent of the specimen geometry, the tangential residual stress values measured are in the range between 20 and $102 \mathrm{MPa}$.

\section{CONCLUSION}

The quench hardening process has been simulated by a finite element method and various case studies have been carried out. The effects of workpiece geometry have been investigated experimentally. The results of computer simulation are highly dependent on the input data used. The convective heat transfer coefficient, which is dependent on bath temperature and quench severity, is the most critical physical property. The assumed value of $h$ is very important to obtain correct results.

The quench bath temperature has an important effect on the final residual stress condition. It affects directly the convective heat transfer coefficient, i.e., cooling rates within the cylinder, internal stress evolution due to thermal gradient and phase transformation during quenching, and therefore, the final 
distribution of the residual stresses.

The geometry of the specimen has a considerable effect on the residual stress state within the quenched $\mathrm{C} 60$ steel parts. The hole diameter and degree of eccentricity affects the cooling rates, and therefore, microstructural evolution in the entire volume. Under the same quenching conditions (from $830^{\circ} \mathrm{C}$ into $60^{\circ} \mathrm{C}$ water), if the ratio of the hole diameter to the outer diameter is greater than $0.3 \mathrm{a}$ transition occurs to the through-hardening case, i.e., the cylinder is fully martensitic. Therefore, the residual stress distribution is reversed, i.e., tensile in the surface and compressive in the center, with associated cracking danger. The cracking occurs when the ratio of the hole diameter to the outer diameter reaches 0.6 . On the other hand, the eccentricity of the hole results in compressive-tensile residual stress transition zones along the circumference. For a constant hole diameter, the eccentricity ratio affects the amount of the surface residual stresses and their distribution along the circumference. The effect of eccentricity starts to decrease when the ratio of the hole diameter to the outer diameter reaches $\mathbf{0 . 5}$. However, the effect of geometry is dependent on the steel type, i.e., there is no such effect in the case of $90 \mathrm{MnCrV8}$ steel after the quench hardening procedure is applied.

\section{ACKNOWLEDGMENT}

The authors are grateful to the Bundesanstalt für Materialprüfung (BAM-Berlin) for conducting X-ray measurements. Stimulating and useful discussions with Dipl.-Phys. W. Schuler (BAM) are gratefully acknowledged.

\section{REFERENCES}

1. H.J. Yu, "Berechnung von Abkühlungs-, Umwandlungs-, Schweiss-, sowie Verformungseigenspannung mit Hilfe der Methode der Finiten Elemente", Dr.-Ing. Dissertation, University of Karlsruhe, 1977.

2. B. Hildenwall, "Prediction of the Residual Stresses Created During Quenching", Ph.-D. Thesis, University of Linkoping, 1979.

3. S. Sjoström, Mat. Sci. Tech., 1, 823-829 (1985).

4. S. Denis, E. Gautier, A. Simon ānd G. Beck, Mat. Sci. Tech., 1, 805-814 (1985).

5. J.-B. Leblond, G. Mottet, J. Devaux ând J.-C. Devaux, Mat. Sci. Tech., 1, 815-822 (1985).

6. R. Schröder, "Untersuchungen zur Spannungs- und Eigenspannungsausbildung beim Abschrecken von Stahlzylindern", Dr.-Ing. Dissertation, University of Karlsruhe, 1985.

7. P. Graja, "Rechnerische und experimentelle Untersuchungen zum Einfluss kontinuerlicher und diskontinuerlicher Wärmbehandlungsverfahren auf die Wärme- and Umwandlungseigen- 
spannungen und Verzüge von un- und niedriglegierten Stahlen", Dr.-Ing. Dissertation, University of Karlsruhe, 1987.

8. T. Inoue, D.-Y. Ju and K. Arimoto, Proceedings of $I^{\text {st }}$ International Conference on Quenching and Control of Distortion, Chicago, Illinois, 22-25 September, 1992; pp. 205-211.

9. Y. Fa-rong and W. Shang-li, Mat. Sci. Tech., 1, 851-856 (1985).

10. C.H. Gür, "Finite Element Modeling and Measurement of Stress Evolution in Quenching Processes", Ph.-D. Thesis, Middle East Technical University, Ankara, 1995.

11. E. Scheil, Arch. Eisenhüttenwes., 12, 565-570 (1935).

12. M. Ortiz and E.P. Popov, Int. J. Num. Meth. Engng., 21, 1561-1576 (1985).

13. D.P. Koistinen and R.E. Marburger, Acta Metall., 7, 59-61 (1959).

14. C.H. Gür, A.E. Tekkaya and W. Schuler, "Effect of boundary conditions and workpiece geometry on residual stresses and microstructure in quenching process", Steel Research, 67 (11), 501-506 (1996).

15. C.H. Gur and A.E. Tekkaya, "Finite element simulation of quench hardening", Steel Research, 67 (7), 298-306 (1996). 